\title{
Die Wiederentdeckung der Buchpastoral
}

\author{
von Walter Friedberger
}

In der Reflexion über Möglichkeiten der Seelsorge geschieht derzeit eine interessante Wiederentdeckung, nämlich die des Buches als Medium des Heilsdienstes. Dieser Vorgang entspricht zunächst einer Notsituation. Die Glaubensvermittlung und Hinführung zum Leben aus dem Glauben in den christlichen Gemeinden gelingen nicht mehr umfassend genug. Nicht nur weil viele Menschen ihr Leben überhaupt und rundherum von der kirchlichen Gemeinschaft abkoppeln, sondern weil viele, vielleicht sogar die Mehrzahl, in einer ausdrücklichen Zugehörigkeit zur Kirche ihr Leben so gestalten, wie sie selbst meinen.

Eine für die Pastoral grundlegende Erkenntnis ist, daß die Kirche mit ihren Verkündigungsmöglichkeiten im Leben der Menschen nicht mehr Regie führt. Vielmehr beziehen die Menschen Informationen über Lebensstile und Lebenschancen aus vielen Quellen und gestalten dies alles in einer mehr oder weniger gekonnten Eigenregie. Diese Situation rückt dem hellsichtigen Theoretiker und Praktiker der Seelsorge das Buch immer mehr in die Mitte seiner Aufmerksamkeit. Er entdeckt, wie wichtig es faktisch für den Menschen ist (die Verbreitungsziffern und die Bestsellerlisten machen dies bekannt) und wie wenig sich Seelsorge erlauben kann, am Buch als Medium der Verkündigung vorbeizudenken.

Wenn man in diesem Zusammenhang von Buch spricht, denkt man an Publikationen mit religiös gediegenem Inhalt, katechetische Hilfsmittel und Traktate zur Gestaltung der Frömmigkeit. Sicherlich ist dies ein wichtiges und unverzichtbares Element der Pastoral. In der Anlage einer Buchpastoral muß man jedoch die Kreise weiter ziehen, und zwar so weit, daß jedes Buch, das menschliches Leben fördern und Impulse zum "wahren" Leben geben kann, davon umschlossen wird. Es geht um das für den Menschen hilfreiche Buch überhaupt.

Für die Verantwortlichen in der Seelsorge ergibt sich in Zusammenarbeit mit Verlegern, Buchhändlern und Schriftstellern als erstes die Frage, woran man gute Bücher erkennt, wie man gute Bücher verbreitet, woran man schädliche Bücher erkennt und wie man vor ihnen bewahrt.

Das kirchliche Leben früherer Jahrzehnte und Jahrhunderte kümmerte sich besonders um die Bewahrung vor "gefährlicher" Literatur. Das ist heute nicht mehr vordringlich.

Nur noch selten ist es in der Seelsorge notwendig, vor bestimmten Büchern zu warnen (es gibt jedoch Fälle, wo man daran nicht vorbeikommt). Im großen und ganzen sollte heutige Seelsorge darauf bedacht sein, die Selbständigkeit des Urteils zu fördern und durch Rat und Angebot guter Literatur lebenswertes Lesen zu fördern.

Die Pastoral muß raten und anbieten. das ist keine leichte Angelegenheit, weil die wenigsten Verantwortlichen in den Pfarreien den Öberblick haben und entsprechende

Dr. Walter Friedberger, 1926 geboren, ist Leiter der Theologischen Fortbildung im Auftrag der Bayerischen Bischofskonferenz in Freising, Lehrbeauftragter für katholische Soziallehre an der Universität Regensburg und Pfarrer in Niederbayern. 
Auswahl nicht treffen können. Dazu braucht es notwendige Hilfe und Unterstützung von seiten der fachkundigen Persönlichkeiten im katholischen Buchhandel oder in diözesanen Einrichtungen.

In der örtlichen Seelsorge erheben sich eine Reihe anderer sehr wichtiger Fragen, die jedoch alle einigermaßen lösbar sind. Da geht es darum, wie in der Pfarrei das Angebot von guter Lektüre organisiert werden kann, so, daß regelmäßig und bei bestimmten Anlässen wirksam die richtigen Bücher zu den richtigen Leuten kommen. Buchpastoral muß aus diesen Gründen stark an den Zielgruppen orientiert werden.

Die etwas schwierige, aber auch lösbare Aufgabe ist die Motivation zum Lesen. Es ist ja notwendig, gewisse Trägheitsmomente, Angstzustände und Anstrengungshindernisse zu überwinden. Genau genommen müssen die Menschen immer wieder die Erfahrung machen können, daß das Lesen bestimmter Bücher ihr eigenes Leben bereichert. Gegenteilige Erfahrung dadurch z. B., daß sie langweilende Bücher in die Hand bekommen, erreicht das Gegenteil.

Viele Seelsorger wenden ein, daß die meisten Menschen unfähig seien zum Lesen eines sogenannten guten Buches. Dem muß man zustimmen und hinzufügen, daß manche gute Bücher kurzweilig und unterhaltsam sind, aber die Eigenart vieler solcher Bücher ihre Langweiligkeit ist. Gewiß gehört auch zur Hinführung zum Lesen, den Menschen zu animieren; eine gewisse Anstrengung durch Konzentration auf sich zu nehmen. Ohne dieses wird man in der Buchpastoral nicht weit kommen. Wichtiger aber ist die Einübung des Lesens und die Erfahrung, daß Lesen Spaß macht oder Spaß machen kann. In den örtlichen Gemeinden und anderen kirchlichen Gruppierungen gibt es dazu eine Reihe von Möglichkeiten. Allein schon die Wiedergabe einer Geschichte aus einem Buch bei der sonntäglichen Predigt oder in der Gruppenstunde kann das Interesse an einem bestimmten Buch wecken.

Jedes gute Buch hat in irgendeiner Weise eine Botschaft für den Menschen parat, eine Botschaft, aus der das ewige Wort Gottes widerhallt. Wer zum Lesen guter Bücher führt, vollzieht in eminenter Weise deshalb einen Heilsdienst. - Es kommt nur darauf an, daß in den Kreisen der für die Seelsorge Verantwortlichen die große Möglichkeit einer "Seelsorge durch das Buch" erkannt wird".

\section{Anmerkung}

1 Zum Ganzen: Walter Friedberger: Leben aus Büchern, herausgegeben vom Verband katholischer Verleger und Buchhändler e. V., Stuttgart 1982. 


\section{SUMMARY}

At present the book is being rediscovered as a means of Salvation. The Church does rot guide the life of mankind anymore with its proclamations, but own information and own initiatives dominate. It is therefore evident that the pastoral ministry may not miss the chance which the book offers, by councelling and offering, supported by the competence of the Catholic book trade and diocesan institutions. The book ministry should be directed at target groups, and should motivate towards reading. Sermons and group meetings offer possibilities to this end. The responsibles ought to learn to make use of them.

\section{RESUME}

Actuellement, on redécouvre le livre comme moyen au service du salut. L'Eglise ne règne plus sur la vie des hommes avec son annonciation. Il est de plus en plus clair que la direction de conscience ne doit pas ignorer la chance de l'annonciation par le livre. La pastorale doit alors deviner et offrir, soutenue par la compétence de la librairie catholique et des institutions diocésaines. Mais la pastorale du livre doit s'orienter à des groups servant de cibles. Et elle doit motiver la lecture. Le sermon ou même la rencontre de groupe doit en offrir les possibilités. Les responsables doivent apprendre à profiter des occasions.

\section{RESUMEN}

Está siendo redescubierto el libro como medio al servicio del mensaje de la salvación. Con la proclamatión de la Palabra, la Iglesia ya no ejerce una función directiva en la vida de los hombres. Dominan la propia información y la auto-orientación. Por ello resulta evidente que la pastoral no debe ignorar las posibilidades que el libro ofrece a la proclamación de la Palabra. La pastoral debe aconsejar y ofrecer, apoyada por la librería católica y las institutiones diocesanas. La pastoral bibliográfica debe orientarse a determinados grupos y motivar a la lectura. La predicación y las reuniones de grupo ofrecen posibilidades es este sentido. Los responsables deben tomarla en serio. 